HUB-EP-99/59

THU-99/35

hep-th/9912225

\title{
BPS Amplitudes, Helicity Supertraces and Membranes in M-Theory
}

\author{
Bernard de Wit ${ }^{1,2}$ a and Dieter Lüst ${ }^{1,3}$ 而 \\ ${ }^{1}$ Institute for Theoretical Physics, University of California, \\ Santa Barbara, CA 93106, USA \\ ${ }^{2}$ Institute for Theoretical Physics, Utrecht University, \\ Princetonplein 5, 3508 TA Utrecht, The Netherlands \\ ${ }^{3}$ Institut für Physik, Humboldt Universität zu Berlin, \\ Invalidenstrasse, D-10115 Berlin, Germany
}

\begin{abstract}
We study BPS dominated loop amplitudes in M-theory on $T^{2}$. For this purpose we generalize the concept of helicity supertraces to nine spacetime dimensions. These traces distinguish between various massive supermultiplets and appear as coefficients in their one-loop contributions to $n$-graviton scattering amplitudes. This can be used to show that only ultrashort BPS multiplets contribute to the $R^{4}$ term in the effective action, which was first computed by Green, Gutperle and Vanhove. There are two inequivalent ultrashort BPS multiplets which describe the Kaluza-Klein states and the wrapped membranes that cover the torus a number of times. From the perspective of the type-II strings they correspond to momentum and winding states and D0 or D1 branes.
\end{abstract}

December 1999

\footnotetext{
${ }^{a}$ bdewit@phys.uu.nl

${ }^{b}$ luest@physik.hu-berlin.de
} 
M-theory is believed to provide a unifying framework of all known superstring theories. Its low-energy limit is described by eleven-dimensional supergravity [1]. The latter theory exhibits nonrenormalizable ultraviolet behavior which will presumably be cured once one includes the additional M-theory degrees of freedom. Upon compactification to lower dimensions, all the duality symmetries of string theory, such as S-, T- and U-duality, should become manifest. However the fundamental, microscopic formulation of M-theory is so far unknown. Matrix theory [2, 3] is one attempt in this direction. Closely related to this is the idea that supermembranes [四] constitute the fundamental degrees of freedom of M-theory. Supermembrane theory may not suffer from the incompleteness of perturbative string theory. Unlike string theory, which has both a string tension as well as a coupling constant, it has no conventional perturbative expansion as its only parameter is the membrane tension $T_{\mathrm{m}}$.

In this paper we consider BPS-dominated amplitudes[? in M-theory compactified on a two-dimensional torus $T^{2}$, or equivalently type-IIA/B superstring theory compactified on $S^{1}$. BPS states play an important role in the computation of loop amplitudes in theories with extended supersymmetry. The masses of the BPS states are often supposed to be exact functions of the moduli so that amplitudes that receive their contributions exclusively from BPS states are also exact, even beyond perturbation theory. Here we will focus on the one-loop four-graviton scattering amplitude which provides the coefficient of the gravitational $R^{4}$ term in the corresponding low-energy effective action. Computing this one-loop amplitude in eleven-dimensional supergravity compactified on $T^{2}$ to nine spacetime dimensions [5, 17, 18, 19, 20], the nonvanishing contribution is associated with the sum over the tower of doubly-charged Kalazu-Klein states which circulate in the nine-dimensional loop.

From a nine-dimensional perspective one can study the one-loop contributions to the $R^{4}$ term coming from a variety of supermultiplets. In order to analyze what kind of massive states can contribute to the loop amplitude, we adopt the concept of helicity supertraces [13, 14, 15] but now extended to nine dimensions. We will then show that the $R^{4}$ terms are exclusively generated by the ultrashort BPS multiplets. As was recently dicussed [16] there are two inequivalent ultrashort multiplets, one corresponding to the Kaluza-Klein states from eleven-dimensional supergravity and the other one corresponding to the Kaluza-Klein states from IIB supergravity. The latter states can be interpreted as the wrapped membrane states of M-theory. Summing over both types of states thus yields the exact answer for M-theory, as other massive supermultiplets cannot contribute. We then show that including the sum

${ }^{c}$ BPS amplitudes in perturbative and nonperturbative string theory have been discussed in many places - see e.g. [6, 7, 8, 9, 10, 11, 12]. 
over the wrapped membrane states (using the same subtraction method as used in [5] for the Kaluza-Klein states on $T^{2}$ ) yields the correct amplitude consistent with T-duality. The $R^{6}$ terms receive contributions from intermediate BPS multiplets but not from long (non-BPS) multiplets, while $R^{8}$ and higher-order terms receive contributions from all supermultiplets

Let us now first generalize the concept of helicity (super)traces for massive states to nine spacetime dimensions where the group of rest-frame rotations is given by $\mathrm{SO}(8)$. Following [14] we define a generating function for the helicity traces by

$$
Z_{\mathbf{r}}(y)=\operatorname{Tr}_{\mathbf{r}}\left(g_{\mathrm{SO}(8)}\right)=\operatorname{Tr}_{\mathbf{r}}(\exp [i \vec{\phi} \cdot \vec{H}])
$$

where the trace and the $\mathrm{SO}(8)$ group element $g_{\mathrm{SO}(8)}$ are defined in a representation r. Obviously the generating function depends only on the group conjugacy classes parametrized by four angles $\phi_{i}$ associated with the torus of the Cartan subalgebra. The $H_{i}$ denote the generators of the Cartan subalgebra in the representation $\mathbf{r}$ and the variables $y_{i}$ are defined by $y_{i}=\exp \left[i \phi_{i} / 2\right]$. The generating functions satisfies the following properties,

$$
\begin{aligned}
Z_{\mathbf{r} \oplus \mathbf{r}^{\prime}}(y) & =Z_{\mathbf{r}}(y)+Z_{\mathbf{r}^{\prime}}(y), \\
Z_{\mathbf{r} \otimes \mathbf{r}^{\prime}}(y) & =Z_{\mathbf{r}}(y) Z_{\mathbf{r}^{\prime}}(y), \\
Z_{\mathbf{r}}(y) & =Z_{\mathbf{r}}\left(y^{-1}\right) \\
Z_{\mathbf{r}}(y=1) & =\operatorname{dim}(\mathbf{r})
\end{aligned}
$$

which are easy to prove. All the above results can readily be generalized to a supertrace for which we will not introduce any new notation. Note, however, that the supertrace for $y_{i}=1$ is equal to the graded trace of the identity and will thus vanish for supermultiplets.

The helicity traces are now $n$-th rank symmetric tensors defined by (no summation over repeated indices)

$$
B_{n}(\mathbf{r})=\left.\frac{1}{2^{n}} y_{i_{1}} \frac{\partial}{\partial y_{i_{1}}} \cdots y_{i_{n}} \frac{\partial}{\partial y_{i_{n}}} Z_{\mathbf{r}}(y)\right|_{y_{i}=1} .
$$

Observe that the trace will vanish for odd values of $n$. In the reduction to four spacetime dimensions, these results coincide with those of [14].

Let us present the generating function for a number of relevant $\mathrm{SO}(8)$ representations,

$$
\begin{aligned}
& Z_{\mathbf{8}_{v}}(y)=\sum_{i}\left(y_{i}^{2}+y_{i}^{-2}\right) \\
& Z_{\boldsymbol{8}_{s}}(y)=\frac{1}{24} \sum_{\substack{i \neq j \neq k \neq l\\
}}\left(y_{i} y_{j} y_{k} y_{l}+6 y_{i} y_{j} y_{k}^{-1} y_{l}^{-1}+y_{i}^{-1} y_{j}^{-1} y_{k}^{-1} y_{l}^{-1}\right), \\
& Z_{\mathbf{8}_{c}}(y)=\frac{1}{6} \sum_{i \neq j \neq k \neq l}\left(y_{i} y_{j} y_{k} y_{l}^{-1}+y_{i} y_{j}^{-1} y_{k}^{-1} y_{l}^{-1}\right)
\end{aligned}
$$




$$
\begin{aligned}
& Z_{\mathbf{2 8}_{0}}(y)=\frac{1}{2} \sum_{i \neq j}\left(y_{i}^{2} y_{j}^{2}+2 y_{i}^{2} y_{j}^{-2}+y_{i}^{-2} y_{j}^{-2}\right)+4, \\
& Z_{\mathbf{3 5}_{0}}(y)=\sum_{i}\left(y_{i}^{4}+y_{i}^{-4}\right)+\frac{1}{2} \sum_{i \neq j}\left(y_{i}^{2} y_{j}^{2}+2 y_{i}^{2} y_{j}^{-2}+y_{i}^{-2} y_{j}^{-2}\right)+3 \text {, } \\
& Z_{\mathbf{3 5}_{0}^{\prime}}(y)=\frac{1}{24} \sum_{i \neq j \neq k \neq l}\left(y_{i}^{2} y_{j}^{2} y_{k}^{2} y_{l}^{2}+6 y_{i}^{2} y_{j}^{2} y_{k}^{-2} y_{l}^{-2}+y_{i}^{-2} y_{j}^{-2} y_{k}^{-2} y_{l}^{-2}\right) \\
& +\frac{1}{2} \sum_{i \neq j}\left(y_{i}^{2} y_{j}^{2}+2 y_{i}^{2} y_{j}^{-2}+y_{i}^{-2} y_{j}^{-2}\right)+3 \text {, } \\
& Z_{\mathbf{3 5}_{0}^{\prime \prime}}(y)=\frac{1}{6} \sum_{i \neq j \neq k \neq l}\left(y_{i}^{2} y_{j}^{2} y_{k}^{2} y_{l}^{-2}+y_{i}^{2} y_{j}^{-2} y_{k}^{-2} y_{l}^{-2}\right) \\
& +\frac{1}{2} \sum_{i \neq j}\left(y_{i}^{2} y_{j}^{2}+2 y_{i}^{2} y_{j}^{-2}+y_{i}^{-2} y_{j}^{-2}\right)+3, \\
& Z_{\mathbf{5 6}_{v}}(y)=\frac{1}{6} \sum_{i \neq j \neq k}\left(y_{i}^{2} y_{j}^{2} y_{k}^{2}+3 y_{i}^{2} y_{j}^{2} y_{k}^{-2}+3 y_{i}^{2} y_{j}^{-2} y_{k}^{-2}+y_{i}^{-2} y_{j}^{-2} y_{k}^{-2}\right) \text {, } \\
& +3 \sum_{i}\left(y_{i}^{2}+y_{i}^{-2}\right) \text {, } \\
& Z_{\mathbf{5 6}_{s}}(y)=\frac{1}{6} \sum_{i \neq j \neq k \neq l}\left(3 y_{i}^{3} y_{j} y_{k} y_{l}^{-1}+y_{i}^{3} y_{j}^{-1} y_{k}^{-1} y_{l}^{-1}+y_{i} y_{j} y_{k} y_{l}^{-3}+3 y_{i} y_{j}^{-1} y_{k}^{-1} y_{l}^{-3}\right) \\
& +\frac{1}{8} \sum_{i \neq j \neq k \neq l}\left(y_{i} y_{j} y_{k} y_{l}+6 y_{i} y_{j} y_{k}^{-1} y_{l}^{-1}+y_{i}^{-1} y_{j}^{-1} y_{k}^{-1} y_{l}^{-1}\right) \text {, } \\
& Z_{\mathbf{5 6}_{c}}(y)=\frac{1}{6} \sum_{i \neq j \neq k \neq l}\left(y_{i}^{3} y_{j} y_{k} y_{l}+3 y_{i}^{3} y_{j} y_{k}^{-1} y_{l}^{-1}+3 y_{i} y_{j} y_{k}^{-1} y_{l}^{-3}+y_{i}^{-1} y_{j}^{-1} y_{k}^{-1} y_{l}^{-3}\right) \\
& +\frac{1}{2} \sum_{i \neq j \neq k \neq l}\left(y_{i} y_{j} y_{k} y_{l}^{-1}+y_{i} y_{j}^{-1} y_{k}^{-1} y_{l}^{-1}\right) \text {. }
\end{aligned}
$$

Note that the three representations $3 \mathbf{5}_{0}, \mathbf{3 5 _ { 0 } ^ { \prime }}$ and $\mathbf{3 5 _ { 0 } ^ { \prime \prime }}$ appear in the square of the $\boldsymbol{8}_{v}, \boldsymbol{8}_{s}$ and $\boldsymbol{8}_{c}$ representations, respectively. These results may for instance be obtained by making use of the weight vectors for these representations, which we have collected in the appendix.

Let us now review the various possible BPS multiplets associated with the ninedimensional $N=2$ supersymmetry algebra with Lorentz invariant central charges [16]. There are three independent charges. Two of them rotate under the action of the the $\mathrm{SO}(2)$ automorphism group, while the other one is invariant. Due to this particular structure of the central charges there exist three types of BPS multiplets (as always we can combine multiplets into larger multiplets with higher spins by assigning spin to the Clifford vacuum, but this does not affect their characterization as BPS states).

First there exist two inequivalent types of ultrashort multiplets of massive $1 / 2$ BPS states, which are annihilated by inequivalent subsets of 16 supercharges. Both multiplets contain $2^{8}=128+128$ states. When the $\mathrm{SO}(2)$ invariant central charge vanishes and the other one has a magnitude equal to the rest mass of the multiplet, the multiplet decomposes as

$$
\left(\mathbf{8}_{v}+\mathbf{8}_{s}\right) \times\left(\mathbf{8}_{v}+\mathbf{8}_{c}\right)=\left[\mathbf{1}_{0}+\mathbf{8}_{v}+\mathbf{2} \mathbf{8}_{0}+35_{0}+56_{v}\right]_{\text {boson }}
$$




$$
+\left[\mathbf{8}_{s}+\mathbf{8}_{c}+\mathbf{5 6} \mathbf{6}_{s}+\mathbf{5 6} \mathbf{6}_{c}\right]_{\text {fermion }} .
$$

This is the multiplet that comprises the Kaluza-Klein states of IIA supergravity compactified on $S^{1}$, which are the momentum states of the compactified IIA string. Therefore this particular multiplet is known as the KKA multiplet. Also the D0branes of the IIA superstring transform according to this multiplet. The second ultrashort multiplet is the KKB multiplet. Now only the noninvariant central charge is different from zero and equal in magnitude to the rest mass. The multiplet decomposes according to

$$
\begin{aligned}
\left(\mathbf{8}_{v}+\mathbf{8}_{c}\right) \times\left(\mathbf{8}_{v}+\mathbf{8}_{c}\right)= & {\left[\mathbf{1}_{0}+\mathbf{1}_{0}+\mathbf{2} \mathbf{8}_{0}+\mathbf{2} \mathbf{8}_{0}+\mathbf{3} \mathbf{5}_{0}+\mathbf{3} \mathbf{5}_{0}^{\prime \prime}\right]_{\text {boson }} } \\
& +\left[\mathbf{8}_{s}+\mathbf{8}_{s}+\mathbf{5} \boldsymbol{6}_{s}+\mathbf{5} \boldsymbol{6}_{s}\right]_{\text {fermion }} .
\end{aligned}
$$

This supermultiplet comprises the Kaluza-Klein states of IIB supergravity compactified on $S^{1}$. Clearly, the BPS states associated with a membrane wrapped around $T^{2}$ in eleven dimensions will also constitute KKB multiplets.

Intermediate multiplets exist of massive 1/4 BPS states annihilated by 8 supercharges. This multiplet carries both types of central charges. The smallest multiplet contains $2^{12}=2^{11}+2^{11}$ states. They do appear in string theory as mixed states that carry both winding and momentum and have a nonzero oscillator number in order to satisfy the mass-shell condition. Hence they carry masses of the order of the string scale. The smallest multiplet associated with the lowest spins decomposes as

$$
\left(\mathbf{8}_{v}+\mathbf{8}_{s}\right) \times\left(\mathbf{8}_{v}+\mathbf{8}_{c}\right) \times\left(\mathbf{8}_{v}+\mathbf{8}_{c}\right)
$$

(or its conjugate).

Finally there are the long (non-BPS) multiplets where all the supercharges act nontrivially. The smallest one comprises $2^{16}$ states and decomposes into

$$
\left(\mathbf{8}_{v}+\mathbf{8}_{s}\right) \times\left(\mathbf{8}_{v}+\mathbf{8}_{s}\right) \times\left(\mathbf{8}_{v}+\mathbf{8}_{c}\right) \times\left(\mathbf{8}_{v}+\mathbf{8}_{c}\right) .
$$

Before proceeding to the determination of the generating functions for the four classes of supermultiplets, let us define these functions for two $N=1$ supermultiplets consisting of $\left(\mathbf{8}_{v}+\mathbf{8}_{s}\right)$ and $\left(\mathbf{8}_{v}+\mathbf{8}_{c}\right)$. Observe that these are precisely the BPS multiplets that one obtains from compactifying the ten-dimensional supersymmetric gauge theory on $S^{1}$. We denote the corresponding generating functions by $Z_{[\mathrm{s}]}$ and $Z_{[\mathrm{c}]}$. They are given byl]

$$
\begin{aligned}
& Z_{[\mathbf{s}]}(y)=Z_{\mathbf{8}_{v}}(y)-Z_{\boldsymbol{8}_{s}}(y), \\
& Z_{[\mathbf{c}]}(y)=Z_{\mathbf{8}_{v}}(y)-Z_{\boldsymbol{8}_{c}}(y),
\end{aligned}
$$

${ }^{d}$ BPS supermultiplets are necessarily complex in order to transform under the central charge. This requires to add an additional factor equal to two in the definition of the generating functions and the helicity traces. We suppress this factor throughout because we eventually sum over the (positive and negative) charges 
and can be calculated from the expressions given in (4). It then follows straightforwardly that the helicity traces generated by $Z_{[\mathrm{s}]}$ and $Z_{[\mathrm{c}]}$ vanish for $n<4$.

From the above result it follows that

$$
\begin{aligned}
Z_{\mathrm{KKA}}(y) & =Z_{\mathbf{r}}(y) Z_{[\mathbf{s}]}(y) Z_{[\mathbf{c}]}(y), \\
Z_{\mathrm{KKB}}(y) & =Z_{\mathbf{r}}(y) Z_{[\mathbf{c}]}^{2}(y), \\
Z_{\mathrm{INTERM}}(y) & =Z_{\mathbf{r}}(y) Z_{[\mathbf{s}]}(y) Z_{[\mathbf{c}]}^{2}(y), \\
Z_{\mathrm{LONG}}(y) & =Z_{\mathbf{r}}(y) Z_{[\mathbf{s}]}^{2}(y) Z_{[\mathbf{c}]}^{2}(y),
\end{aligned}
$$

where $\mathbf{r}$ denotes the spin representation of the Clifford vacuum. This suffices to show that the helicity supertraces $B_{n}$ are vanishing for KKA and KKB multiplets whenever $n<8$, for intermediate multiplets whenever $n<12$ and for long multiplets whenever $n<16$. This result has important implications for the one-loop graviton amplitudes in type-II string theories, as we will show below (obviously there is a corresponding result for $N=1$ theories pertaining to the one-loop gauge field amplitudes).

Subsequently we review the spectrum of the BPS states of M-theory compactified to nine spacetime dimensions on $T^{2}$ [23, 24, 16]. The BPS mass formula based on the supersymmetry algebra with a membrane winding charge, takes the form

$$
M_{q_{1}, q_{2}, p}=\frac{1}{\sqrt{A \tau_{2}}}\left|q_{1}-\tau q_{2}\right|+T_{\mathrm{m}} A|p| .
$$

The ultrashort BPS multiplets are either given by Kalazu-Klein states with momentum numbers $\left(q_{1}, q_{2}\right)$ along the two periods of $T^{2}$ or by wrapped membranes which cover the torus $p$ times. The Kaluza-Klein charges $\left(q_{1}, q_{2}\right)$ transform as doublets under the $S O(2)$ automorphism group of the supersymmetry algebra; the charge associated with the wrapped membrane states is invariant under this group. In (11) $A$ denotes the volume of $T^{2}$, measured with respect to the 11-dimensional metric, $\tau=\tau_{1}+i \tau_{2}$ denotes the complex structure of $T^{2}$ and $T_{\mathrm{m}}$ denotes the membrane tension.

The BPS mass formula (11) can be interpreted in the context of type-II superstrings compactified on $S^{1}$. For this purpose let us recall the well-known relations between the string and M-theory parameters [25, 26]. For simplicity we set $T_{\mathrm{m}}=1$ and assume that the M-theory metric is diagonal and has the form $G_{i j}=\operatorname{diag}\left(r_{9}^{2}, r_{10}^{2}\right)$, so that $A=r_{9} r_{10}, \tau_{1}=0$ and $\tau_{2}=r_{9} / r_{10}$. The ten-dimensional IIA string coupling and the nine-dimensional radius of type-IIA on $S^{1}$ (in the string frame) are related to the M-theory parameters by

$$
g_{10}^{A}=r_{10}^{3 / 2}, \quad R_{9}^{A}=r_{9} \sqrt{r_{10}} .
$$

Using T-duality between IIA and IIB in nine dimensions we can express the corresponding IIB parameters in the following way (everything measured with respect to 
the string metric in units of the string scale $\left.1 / \sqrt{\alpha^{\prime}}\right)$,

$$
R_{9}^{B}=\frac{1}{R_{9}^{A}}=\frac{1}{r_{9} \sqrt{r_{10}}}, \quad g_{10}^{B}=\frac{1}{\tau_{2}}=\frac{r_{10}}{r_{9}} .
$$

Subsequently we can express the BPS mass formula (11) in the string frame in terms of IIA and IIB string theory variables, respectively,

$$
M_{q_{1}, q_{2}, p}=\left|\frac{q_{1}}{R_{9}^{A}}+\frac{q_{2}}{g_{10}^{A}}\right|+|p| R_{9}^{A}=\left|q_{1} R_{9}^{B}+q_{2} \frac{R_{9}^{B}}{g_{10}^{B}}\right|+\frac{|p|}{R_{9}^{B}} .
$$

Therefore from the perspective of the IIA string theory, $q_{1}$ is the IIA Kaluza-Klein momentum number, while $q_{2}$ is the D0-brane charge. The M-theory membrane wrapping number $p$ becomes the perturbative winding number in the IIA string. On the other hand, from the IIB perspective, $q_{1}$ and $q_{2}$ are the winding numbers of the elemenary string and of the solitonic D1 string, and the membrane wrapping number $p$ is the IIB Kaluza-Klein momentum. The IIB strong-weak coupling Sduality interchanges the elementary strings with the D1 strings: $q_{1} \leftrightarrow q_{2}$. Under the IIA/B duality, $q_{1} \leftrightarrow p$, the IIA/IIB mass formulas are interchanged provided one interchanges the D0 with the D1 states. Clearly the presence of the wrapped membranes is essential, as they correspond either to the IIA winding states or to the IIB momentum states, respectively.

In (perturbative) type-II string theory the various BPS states emerge as follows. Ultrashort BPS states, which preserve 16 of the total 32 supersymmetries, must be $1 / 2$ BPS states both with respect to the left-moving $N=1$ and also with respect to the right-moving $N=1$ supersymmetry algebra. Therefore these states are not allowed to carry any oscillator excitations, i.e. $N_{L}=N_{R}=0$. Hence their $\mathrm{SO}(8)$ helicities are entirely determined by the bosonic and fermionic groundstates, $\left(\mathbf{8}_{v}+\boldsymbol{8}_{s(c)}\right)_{L} \times\left(\boldsymbol{8}_{v}+\mathbf{8}_{c}\right)_{R}$, leading to the decomposition in (5) and (6) for the two ultrashort multiplets. Using the string level matching condition, $p_{L}^{2}=p_{R}^{2}$, where $p_{L, R}=q_{1} / R_{9}^{A} \pm p R_{9}^{A}$ for IIA, and similarly for IIB, it follows that the ultrashort BPS states can carry either nonvanishing KKA quantum numbers $q_{i}$ or non-vanishing KKB quantum numbers $p$, but not both.

The intermediate, $1 / 4$ BPS states are short $1 / 2$ BPS multiplets with respect to the left-moving $N=1$ supersymmetry algebra but are long multiplets with respect to the right-moving $N=1$ supersymmetry algebra (or vice versa). Therefore they require $N_{L}=0$ and $N_{R}$ arbitrary. The level matching condition $p_{L}^{2}=p_{R}^{2}+2 N_{R}$ now tells us that intermediate multiplets must have both winding and momentum and tus carry both type of charges. For $N_{R}=1$ we have precisely the decomposition (17), while for higher $N_{R}$ we have intermediate BPS multiplets of higher spin. Finally the long multiplets have $N_{L}, N_{R} \neq 0$. The smallest long multiplet with $N_{L}=N_{R}=1$ has precisely the decomposition (8) and others will have higher spins. 
After this perusal of the various supermultiplets from complementary viewpoints, we briefly discuss the helicity generating partition function of M-theory on $T^{2}$. For string theory it has been argued [15] that this partition function is related to the string amplitudes for multi-graviton scattering which we will turn to shortly. Hence we start from the corresponding expression for the perturbative type-II string in nine dimensions,

$$
Z(\phi, \bar{\phi} ; q, \bar{q})=\operatorname{Str}\left[q^{L_{0}} \bar{q}^{\bar{L}_{0}} e^{i \phi_{i} H_{i}+i \bar{\phi}_{i} \bar{H}_{i}}\right] .
$$

Here $q$ and $\bar{q}$ are related to the world-sheet modular parameter in the usual way. The expression for $Z(\phi, \bar{\phi} ; q, \bar{q})$ can be written as

$$
\begin{aligned}
& Z(\phi, \bar{\phi} ; q, \bar{q}) \sim \frac{1}{|\eta(q)|^{8}} \Gamma_{1,1}(q, \bar{q}) \\
& \quad \times\left[-\prod_{i=1}^{4} \theta_{1}\left(\phi_{i}, q\right)+\prod_{i=1}^{4} \theta_{2}\left(\phi_{i}, q\right)-\prod_{i=1}^{4} \theta_{3}\left(\phi_{i}, q\right)+\prod_{i=1}^{4} \theta_{4}\left(\phi_{i}, q\right)\right] \\
& \quad \times\left[\operatorname{sgn}\left(p_{L} p_{R}\right) \prod_{i=1}^{4} \theta_{1}\left(\bar{\phi}_{i}, \bar{q}\right)+\prod_{i=1}^{4} \theta_{2}\left(\bar{\phi}_{i}, \bar{q}\right)-\prod_{i=1}^{4} \theta_{3}\left(\bar{\phi}_{i}, \bar{q}\right)+\prod_{i=1}^{4} \theta_{4}\left(\bar{\phi}_{i}, \bar{q}\right)\right],
\end{aligned}
$$

where

$$
\Gamma_{1,1}(q, \bar{q})=\sum_{p_{L}, p_{R}} q^{p_{L}^{2} / 2} \bar{q}^{p_{R}^{2} / 2}
$$

Note that in $Z(\phi, \bar{\phi} ; q, \bar{q})$ we have neglected the contributions from the bosonic oscillators (so that we will not discuss the issue of the modular transformation properties of $Z$ ). The products of $\theta$-functions originate from the sum over spin structures and encode the helicities associated with the fermionic zero modes; in particular we draw attention to the presence of the sign factor $\operatorname{sgn}\left(p_{L} p_{R}\right)=\operatorname{sgn}\left(q_{1}^{2} /\left(R_{9}^{A}\right)^{2}-p^{2}\left(R_{9}^{A}\right)^{2}\right)$ in front of the $\theta_{1}\left(\bar{\phi}_{i}, \bar{q}\right)$ functions, which is required in order to correctly account for the different helicities of the momentum and winding states [16]. Obviously the lattice summation in (17) acts on this sign factor as well. Owing to the sign factor, which is +1 for IIA momentum states and IIB winding states, respectively, and -1 for IIA winding states and IIB momentum states, respectively, the partition function (17) tends to the correct expression for IIA string theory in the decompactification limit $R_{9}^{A} \rightarrow \infty$ and for IIB string theory in the limit $R_{9}^{A} \rightarrow 0$.

An intriguing question is whether one can extend the sum over the perturbative momentum and winding string states, by including the nonperturbative BPS states of M-theory with masses as given in (11). By using the $\mathrm{SO}(2)$ symmetry among the Kaluza-Klein states with momentum numbers $q_{1}$ and $q_{2}$, we are led to postulate that the same split into left- and right-moving masses $L_{0}$ and $\bar{L}_{0}$ as in string theory will also hold for compactified M-theory, i.e., we replace $p_{L}$ and $p_{R}$ in the above expression by

$$
\frac{p_{L, R}}{\sqrt{2}}=\frac{q_{1}-\tau q_{2}}{\sqrt{A \tau_{2}}} \pm A T_{\mathrm{m}} p .
$$


Then the M-theory BPS sum takes the following form:

$$
\Gamma_{1,1}(q, \bar{q})=\sum_{q_{1}, q_{2}, p} q^{\left|\frac{q_{1}-\tau q_{2}}{\sqrt{A \tau_{2}}}+A T_{\mathrm{m}} p\right|^{2}} \bar{q}^{\left|\frac{q_{1}-\tau q_{2}}{\sqrt{A \tau_{2}}}-A T_{\mathrm{m}} p\right|^{2}} .
$$

However, $\Gamma_{1,1}$ does not depend on the angles and therefore plays only a minor role on what follows. Clearly, apart from $\Gamma_{1,1}$, the expression (17) factorizes in a holomorphic and an anti-holomorphic part. The appearance of the sum over the powers of theta-functions, $\prod_{i=1}^{4} \theta_{a}\left(\phi_{i}\right)$, is of crucial importance for what follows. Using the Riemann identity the $\theta$-functions, we can rewrite (17) in the following way:

$$
Z(\phi, \bar{\phi} ; q, \bar{q})=\frac{1}{|\eta(q)|^{8}} \Gamma_{1,1}(q, \bar{q}) \prod_{i=1}^{4} \theta_{1}\left(\phi_{i}^{\prime} / 2, q\right) \theta_{1}\left(\bar{\phi}_{i}^{\prime} / 2, \bar{q}\right),
$$

where

$$
\begin{aligned}
\phi_{1}^{\prime}=-\phi_{1}+\phi_{2}+\phi_{3}+\phi_{4}, & \bar{\phi}_{1}^{\prime}=\operatorname{sgn}\left(p_{L} p_{R}\right) \bar{\phi}_{1}+\bar{\phi}_{2}+\bar{\phi}_{3}+\bar{\phi}_{4}, \\
\phi_{2}^{\prime}=-\phi_{1}+\phi_{2}-\phi_{3}-\phi_{4}, & \bar{\phi}_{2}^{\prime}=\operatorname{sgn}\left(p_{L} p_{R}\right) \bar{\phi}_{1}+\bar{\phi}_{2}-\bar{\phi}_{3}-\bar{\phi}_{4}, \\
\phi_{3}^{\prime}=-\phi_{1}-\phi_{2}+\phi_{3}-\phi_{4}, & \bar{\phi}_{3}^{\prime}=\operatorname{sgn}\left(p_{L} p_{R}\right) \bar{\phi}_{1}-\bar{\phi}_{2}+\bar{\phi}_{3}-\bar{\phi}_{4}, \\
\phi_{4}^{\prime}=-\phi_{1}-\phi_{2}-\phi_{3}+\phi_{4}, & \bar{\phi}_{4}^{\prime}=\operatorname{sgn}\left(p_{L} p_{R}\right) \bar{\phi}_{1}-\bar{\phi}_{2}-\bar{\phi}_{3}+\bar{\phi}_{4} .
\end{aligned}
$$

The holomorphic part of the helicity generating partition function is the direct string generalization of the field theory generating function $Z_{\mathbf{8}_{v}}(y)-Z_{\mathbf{8}_{c}}(y)$, discussed before, where in the string case we deal not only with the graded sum over the vector $\mathbf{8}_{v}$ and the spinor $\mathbf{8}_{c}$ ground states, but also with the graded sum over the whole weight lattice related to these two $S O(8)$ representations. This sum vanishes for $\phi_{i}=0$ due to space-time supersymmetry. The corresponding helicity supertraces are given by the multiple derivatives of the partition function, after setting $\phi_{i}=\bar{\phi}_{i}$, i.e.,

$$
B_{2 n}(q)=\left.(-1)^{n} \frac{\partial}{\partial \phi_{i_{1}}} \cdots \frac{\partial}{\partial \phi_{i_{2 n}}} Z\left(\phi_{i}, \phi_{i} ; q, \bar{q}\right)\right|_{\phi_{i}=0} .
$$

Because $\theta_{1}(0, q)=0$ and $\partial \theta_{1}(\phi, q) /\left.\partial \phi\right|_{\phi=0}=-2 \pi \eta(q)^{3}$, we need at least four powers of $\partial / \partial \phi_{i}$ and four powers of $\partial / \partial \bar{\phi}_{i}$ in order to get a nonvanishing result, in agreement with the previous discussion. For example, $B_{8}$ can only get contributions from ultrashort KKA or KKB multiplets, while $B_{12}$ receives contributions from both ultrashort and intermediate supermultiplets.

Now we turn to the evaluation of the $n$-graviton amplitudes, i.e. we consider one-loop string amplitudes with $n$ insertions of graviton vertex operators. In the zero-ghost picture, the graviton vertex operator has the form,

$$
G_{m n} \sim\left(\partial X_{m}+i p_{m} \sum_{i=1}^{4} \psi_{i} \psi_{-i}\right)\left(\bar{\partial} \bar{X}_{n}+i p_{n} \sum_{i=1}^{4} \bar{\psi}_{i} \bar{\psi}_{-i}\right) \mathrm{e}^{i p \cdot X},
$$


Hence the graviton amplitude can be expanded in terms of powers of the operators $\psi_{i} \psi_{-i}$ and $\bar{\psi}_{i} \bar{\psi}_{-i}$ which are the Cartan subalgebra currents of $\mathrm{SO}(8)$ in the left- and right-moving sector generated by the world-sheet fermions. Their charges $H_{i}=(2 \pi i)^{-1} \oint \psi_{i} \psi_{-i}$ and $\bar{H}_{i}=(2 \pi i)^{-1} \oint \bar{\psi}_{i} \bar{\psi}_{-i}$ are the Cartan algebra generators of $\mathrm{SO}(8)$. Furthermore the amplitude factorizes into contributions from the left- and the right-moving sector. Each contribution thus decomposes into a linear combination of the helicity supertraces that are generated by the functions (9). Because the number of gravitons is equal to the highest power of of helicity operators $H_{i}$ and $\bar{H}_{i}$, the $n$-graviton amplitude is proportional to $B_{2 n}$ and lower helicity supertraces. This proves that the four-graviton amplitude must be proportional to the helicity supertrace $B_{8}$ and will thus receive constributions from only ultrashort BPS multiplets. Likewise the six-graviton amplitude will receive contributions from both ultrashort and intermediate BPS multiplets. The contribution from the latter contains a term proportional to $B_{12}$. For eight and more gravitons all supermultiplets will in principle contribute.

Having established these results, we briefly consider the $R^{4}$ terms in more detail. Here we cast the calculation in terms of nine-dimensional field theory, where the relevant amplitude has the structure of a box diagram in massive $\varphi^{3}$ theory in nine spacetime dimensions. In view of our previous results it suffices to restrict ourselves to contributions from the KKA and KKB states. Since we evaluate the coefficient of the $R^{4}$ term at zero momentum, such an integral takes the form

$$
\frac{1}{(2 \pi)^{9}} \int \frac{d^{9} q}{\left(q^{2}+M^{2}\right)^{4}}=\frac{1}{6} \frac{1}{(4 \pi)^{9 / 2}} \int_{0}^{\infty} d t t^{-3 / 2} \mathrm{e}^{-t M^{2}} .
$$

Observe that this integral has a linear ultraviolet divergence, which reflects itself in the singular behaviour of the integrand at $t=0$. The integral has the dimension of a mass, which is appropriate for a coupling constant of $R^{4}$ in nine spacetime dimensions. We will now use (24) and the BPS mass formula (11) and determine the contributions from both the KKA and the KKB states. For the former we obtain

$$
\begin{aligned}
A_{4}^{\mathrm{KKA}} & =\frac{1}{6} \frac{1}{(4 \pi)^{9 / 2}} \int_{0}^{\infty} d t t^{-3 / 2} \sum_{q_{1}, q_{2}} \mathrm{e}^{-t\left(A \tau_{2}\right)^{-1}\left|q_{1}-\tau q_{2}\right|^{2}} \\
& =\frac{1}{3} \frac{A^{-1 / 2}}{(4 \pi)^{5}} \int_{0}^{\infty} d t t^{1 / 2} \sum_{q_{1}^{\prime}, q_{2}^{\prime}} \mathrm{e}^{-\pi t \tau_{2}^{-1}\left|q_{1}^{\prime}+\tau q_{2}^{\prime}\right|^{2}} \\
& =\frac{2}{3} \frac{A^{-1 / 2}}{(4 \pi)^{6}} f(\tau, \bar{\tau})
\end{aligned}
$$

where the modular function $f(\tau, \bar{\tau})$ is defined by [5]

$$
f(\tau, \bar{\tau})=\sum_{\left(q_{1}^{\prime}, q_{2}^{\prime}\right) \neq(0,0)} \frac{\tau_{2}^{3 / 2}}{\left|q_{1}^{\prime}+\tau q_{2}^{\prime}\right|^{3}} .
$$


In the second line of 25) we performed a Poisson resummation and changed the integration variable $t \rightarrow \pi A / t$. Observe that the ultraviolet divergence is now associated with the upper integration boundary and is only present for the term with $q_{1}^{\prime}=q_{2}^{\prime}=0$. This contribution was dropped in the third line.

Likewise, we obtain for the KKB states,

$$
\begin{aligned}
A_{4}^{\mathrm{KKB}} & =\frac{1}{6} \frac{1}{(4 \pi)^{9 / 2}} \int_{0}^{\infty} d t t^{-3 / 2} \sum_{p} \mathrm{e}^{-t T_{\mathrm{m}}^{2} A^{2} p^{2}} \\
& =\frac{1}{3} \frac{T_{\mathrm{m}} A}{(4 \pi)^{5}} \int_{0}^{\infty} d t \sum_{p^{\prime}} \mathrm{e}^{-\pi t p^{\prime 2}} \\
& =\frac{4}{3} \frac{T_{\mathrm{m}} A}{(4 \pi)^{6}} \sum_{p^{\prime} \neq 0} \frac{1}{p^{\prime 2}}
\end{aligned}
$$

Again we performed a Poisson resummation and changed the integration variable $t \rightarrow \pi / t\left(T_{\mathrm{m}} A\right)^{2}$; and we dropped the ultraviolet divergence associated with $p^{\prime}=0$ in the third line. The sum over $p^{\prime}$ in the third line is equal to $2 \zeta(2)=\pi^{2} / 3$. We return to the issue of ultraviolent divergences shortly.

Combining the results (25) and (27) gives rise to

$$
A_{4}^{\mathrm{KKA}+\mathrm{KKB}}=\frac{2}{3} \frac{1}{(4 \pi)^{6}}\left[A^{-1 / 2} f(\tau, \bar{\tau})+\frac{2}{3} \pi^{2} T_{\mathrm{m}} A\right] .
$$

This result is invariant under the IIB S-duality symmetry $\tau \rightarrow(a \tau+b) /(c \tau+d)$. The contribution from the KKB states is such that the result is compatible with T-duality of type-II string theory.

Let us consider the two possible decompactification limits to the ten-dimensional IIA/B theories. Here we need the result that, for $\tau_{1}=0$ and $\tau_{2}$ large, the function $f(\tau, \bar{\tau})$ has the form

$$
f(\tau, \bar{\tau})=2 \zeta(3) \tau_{2}^{3 / 2}+\frac{2}{3} \pi^{2} \tau_{2}^{-1 / 2}+\cdots
$$

up to terms that are exponentially suppressed. We can now use the relations between string and M-theory parameters and rewrite the expression (28) as

$$
A_{4}^{\mathrm{KKA}+\mathrm{KKB}}=\frac{4}{3} \frac{\sqrt{r_{10}} R_{9}^{B}}{(4 \pi)^{6}}\left[\left(\frac{\zeta(3)}{\left(g_{10}^{B}\right)^{2}}+\frac{1}{3} \pi^{2}+\cdots\right)+\frac{1}{3} \pi^{2} \frac{1}{\left(R_{9}^{B}\right)^{2}}\right] .
$$

For large $R_{9}^{B}$ the last term vanishes while the first two terms yield the tree and the one-loop contributions to the $R^{4}$ term, and the ellipses denote exponentially suppressed terms in $\left(g_{10}^{B}\right)^{-2}$, which correspond to nonperturbative IIB D-instanton contributions. (The factor $\sqrt{r_{10}}$ is related to the fact that we expressed the KKA and KKB masses in the M-theory frame; in the string frame this factor disappears.)

In the IIA decompactification limit $R_{9}^{A} \rightarrow \infty$ we obtain

$$
A_{4}^{\mathrm{KKA}+\mathrm{KKB}}=\frac{4}{3} \frac{\sqrt{r_{10}} R_{9}^{A}}{(4 \pi)^{6}}\left[\left(\frac{\zeta(3)}{\left(g_{10}^{A}\right)^{2}}+\frac{1}{3} \pi^{2} \frac{1}{\left(R_{9}^{A}\right)^{2}}+\cdots\right)+\frac{1}{3} \pi^{2}\right] .
$$


Now the ellipses denote terms that are exponentially suppressed in $\left(R_{9}^{A} / g_{10}^{A}\right)^{2}$. Dropping these terms as well as the second term term which all vanish in the decompactification limit, we are left with the IIA string tree and one-loop contribution to $R^{4}$ (the latter originated from the KKB multiplets). Hence perturbative T-duality is manifest is manifest in the results

It is an intriguing question why the subtraction method based on Poisson resummation and the subsequent subtraction of the $q_{1}^{\prime}=q_{2}^{\prime}=0$ and $p^{\prime}=0$ terms leads to the correct result. A puzzling feature of the calculation is that the sum over the KKA and the sum over the KKB states both include the massless states, which seems to make no sense. Correcting for this requires to subtract an infinite term which could in principle be cancelled against the positive infinite contributions of the massive BPS states. However, why the cut-off should be fine-tuned such that this cancellation takes place remains a mystery. Within the context of ninedimensional supersymmetry there are no other states that could possibly cancel the infinite terms. The coefficient of the $R^{4}$ term is not generically finite, which reflects itself in the fact that the KKA contributions disappear in the decompactification limit to eleven dimensions, $A \rightarrow \infty$, which is somewhat counterintuitive. We stress that supersymmetry does not seem to be relevant for obtaining finite results, in view of the fact that the supertraces for the KKA and KKB multiplets do not vanish, and no cancellation seems to arise between the inifinite contributions from the two types of multiplets.

\section{Acknowledgements}

We thank Mohab Abou-Zeid, Elias Kiritsis, Wolfgang Lerche, Hermann Nicolai and Kostas Skenderis for clarifying discussions. This research was supported in part by the National Science Foundation under Grant No. PHY94-07194 through the Institute for Theoretical Physics in Santa Barbara. We thank the institute for the hospitality extended to us during this work. The research was also supported by the European Commission TMR Program under contract ERBFMRX-CT96-0045, in which Humboldt University at Berlin and Utrecht University are associated.

\section{Appendix}

For the convenience of the reader we present the weight vectors for a number of $\mathrm{SO}(8)$ representations which can be used in the construction of the generating functions (4). To each of the weight vectors one must add all possible permutations. In certain cases the number of minus signs must be even or odd. This is indicated by, e.g. $[+20$; even], implying that 20 independent permutations should be added with an even number of minus signs. The subscripts $0, v, s$ and $c$ refer to the different 
conjugacy classes of weight vectors.

$$
\begin{aligned}
& \mathbf{8}_{v}: \quad \vec{\lambda}=( \pm 1,0,0,0) \quad[+6] ; \\
& \mathbf{8}_{s}: \quad \vec{\lambda}=\left( \pm \frac{1}{2}, \pm \frac{1}{2}, \pm \frac{1}{2}, \pm \frac{1}{2}\right) \quad \text { [even]; } \\
& \mathbf{8}_{c}: \quad \vec{\lambda}=\left( \pm \frac{1}{2}, \pm \frac{1}{2}, \pm \frac{1}{2}, \pm \frac{1}{2}\right) \quad[\text { odd }] ; \\
& \mathbf{2 8}_{0}: \quad \vec{\lambda}=( \pm 1, \pm 1,0,0) \quad[+20], \quad \vec{\lambda}_{1,2,3,4}^{\prime}=(0,0,0,0) \text {; } \\
& 35_{0}: \quad \vec{\lambda}=( \pm 2,0,0,0) \quad[+6], \quad \vec{\lambda}^{\prime}=( \pm 1, \pm 1,0,0) \quad[+20] \text {, } \\
& \vec{\lambda}_{1,2,3}^{\prime \prime}=(0,0,0,0) \text {; } \\
& \text { 35 } 5_{0}^{\prime}: \quad \vec{\lambda}=( \pm 1, \pm 1, \pm 1, \pm 1) \quad \text { [even], } \\
& \vec{\lambda}^{\prime}=( \pm 1, \pm 1,0,0) \quad[+20], \quad \vec{\lambda}_{1,2,3}^{\prime \prime}=(0,0,0,0) \text {; } \\
& 35_{0}^{\prime \prime}: \quad \vec{\lambda}=( \pm 1, \pm 1, \pm 1, \pm 1) \quad \text { [odd] } \\
& \vec{\lambda}^{\prime}=( \pm 1, \pm 1,0,0) \quad[+20], \quad \vec{\lambda}_{1,2,3}^{\prime \prime}=(0,0,0,0) \text {; } \\
& \mathbf{5 6}_{v}: \quad \vec{\lambda}=( \pm 1, \pm 1, \pm 1,0) \quad[+24], \quad \vec{\lambda}_{1,2,3}^{\prime}=( \pm 1,0,0,0) \quad[+6] ; \\
& \mathbf{5 6}_{s}: \quad \vec{\lambda}=\left( \pm \frac{3}{2}, \pm \frac{1}{2}, \pm \frac{1}{2}, \pm \frac{1}{2}\right) \quad[+3 \text {; odd }] \text {, } \\
& \vec{\lambda}_{1,2,3}^{\prime}=\left( \pm \frac{1}{2}, \pm \frac{1}{2}, \pm \frac{1}{2}, \pm \frac{1}{2}\right) \quad[\text { even] } \\
& \mathbf{5 6}_{c}: \quad \vec{\lambda}=\left( \pm \frac{3}{2}, \pm \frac{1}{2}, \pm \frac{1}{2}, \pm \frac{1}{2}\right) \quad[+3 ; \text { even }] \text {, } \\
& \vec{\lambda}_{1,2,3}^{\prime}=\left( \pm \frac{1}{2}, \pm \frac{1}{2}, \pm \frac{1}{2}, \pm \frac{1}{2}\right) \quad[\text { odd }] \text {. }
\end{aligned}
$$

\section{References}

[1] E. Cremmer, B. Julia and J. Scherk, Supergravity Theory in Eleven Dimensions, Phys. Lett. 76B (1978) 409.

[2] B. de Wit, J. Hoppe and H. Nicolai, On the Quantum Mechanics of Supermembranes, Nucl. Phys. B305 (1988) 545.

[3] T. Banks, W. Fischler, S.H. Shenker and L. Susskind, M-Theory as a Matrix Model: a Conjecture, Phys. Rev. D55 (1997) 5112, hep-th/9610043.

[4] E. Bergshoeff, E. Sezgin and P.K. Townsend, Supermembranes and ElevenDimensional Supergravity, Phys. Lett. B189 (1987) 75.

[5] M.B. Green, M. Gutperle and P. Vanhove, One Loop in Eleven Dimensions, Phys. Lett. B409 (1997) 177, hep-th/9706175.

[6] L. Dixon, V. Kaplunovsky and J. Louis, Moduli Dependence of String Loop Corrections to Gauge Coupling Constants, Nucl. Phys. B355 (1991) 649. 
[7] S. Ferrara, C. Kounnas, D. Lüst and F. Zwirner, Duality Invariant Partition Functions and Automorphic Superpotentials for (2,2) String Compactifications, Nucl. Phys. B365 (1991) 431.

[8] J. Harvey and G. Moore, Algebras, BPS States and Strings, Nucl. Phys. B463 (1996) 315, hep-th/9510182.

[9] G. Lopes Cardoso, G. Curio, D. Lüst, T. Mohaupt and S.-J. Rey, BPS Spectra and Nonperturbative Gravitational Couplings in $N=2, N=4$ Supersymmetric String Theories, Nucl. Phys. 464 (1996) 18, hep-th/9512129.

[10] J. Harvey and G. Moore, Five-Brane Instantons and $R^{2}$ Couplings in $N=4$ String Theory, Phys. Rev. D57 (1998) 2323, hep-th/9610237.

[11] A. Gregori, E. Kiritsis, C. Kounnas, N.A. Obers, P.M. Petropoulos and B. Pioline, $R^{2}$ Corrections and Nonperturbative Dualities of $N=4$ String Ground States, Nucl. Phys. B510 (1998) 423, hep-th/9708062.

[12] W. Lerche and S. Stieberger, 1/4 BPS States and Nonperturbative Couplings in $N=4$ String Theories, hep-th/9907133.

[13] S. Ferrara, C.A. Savoy and L. Girardello, Spin Sum Rules in Extended Supersymmetry, Phys. Lett. 105B (1981) 363.

[14] C. Bachas and E. Kiritsis, $F^{4}$ Terms in N=4 String Vacua, hep-th/9611205.

[15] E. Kiritsis, Introduction to Non-Perturbative String Theory, hep-th/9708130.

[16] M. Abou-Zeid, B. de Wit, D. Lüst and H. Nicolai, Space-Time Supersymmetry, IIA/B Duality and M-Theory, Phys. Lett. 466B (1999) 144, hep-th/9908169.

[17] M.B. Green and M. Gutperle, Effects of D-Intantons, Nucl. Phys. B498 (1997) 195, hep-th/9701093.

[18] M.B. Green and P. Vanhove, D-Instantons, Strings and M-Theory, Phys. Lett. B408 (1997) 122, hep-th/9704145.

[19] M.B. Green, H. Kwon and P. Vanhove, Two Loops in Eleven Dimensions, hepth/9910055.

[20] M.B. Green and P. Vanhove, The Low Energy Expansion of the One-Loop Type II Superstring Amplitude, hep-th/9910056.

[21] M. Dine, P. Huet and N. Seiberg, Large and Small Radius in String Theory, Nucl. Phys. B322 (1989) 301. 
[22] J. Dai, R. G. Leigh and J. Polchinski, New Connections Between String Theories, Mod. Phys. Lett. A4 (1989) 2073.

[23] J. H. Schwarz, Superstring Dualities, Nucl. Phys. Proc. Suppl. 49 (1996) 183, hep-th/9509148.

[24] J. Russo and A. Tseytlin, Waves, Boosted Branes and BPS States in M-Theory, Nucl. Phys. B490 (1997) 121, hep-th/9611047.

[25] E. Witten, String Theory Dynamics in Various Dimensions, Nucl. Phys. B443 (1995) 85, hep-th/9503124.

[26] J. H. Schwarz, An SL(2,Z) Multiplet of Type-II Superstrings, Phys. Lett. B360 (1995) 13, Erratum: ibid, B364 (1995) 252, hep-th/9508143. 